\title{
Effects of climate, objective function and sample size on global sensitivity in a SWAT Model
}

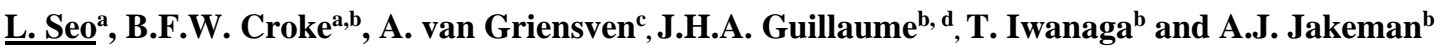 \\ ${ }^{a}$ Mathematical Sciences Institute, The Australian National University, Canberra ACT 0200, Australia \\ ${ }^{b}$ Integrated Catchment Assessment and Management (iCAM) Centre, The Fenner School of Environment and \\ Society, The Australian National University, Canberra ACT 0200, Australia \\ ${ }^{\mathrm{c}}$ Department of Hydrology and Hydraulic Engineering, Earth System Sciences Group, Vrije Universiteit \\ Brussel (VUB), Brussels, Belgium \\ ${ }^{\mathrm{d}}$ Water \& Development Research Group (WDRG)Aalto University, Finland \\ Email:lynn.seo@anu.edu.au
}

\begin{abstract}
Environmental models typically possess large uncertainty due to contributions from model structure, assumptions, parameterization, and data errors, not to mention lack of consideration of problem framing and the associated choice and justification of objective function. Sensitivity analysis (SA) is a fundamental tools to help identify uncertainties relevant to the modelling objectives. It provides information on the impact on model outputs from inputs and parameters and can contribute to simplifying models to make them more identifiable. However, sensitivity analysis can produce different results in accordance with several sources, such as input forcing, objective function, and the sampling undertaken.
\end{abstract}

The Sobol' method of SA is applied here to the Soil and Water Assessment Tool (SWAT). The method is based on variance decomposition, is categorized as a global sensitivity analysis (GSA) and is known to be model independent. It is able to handle non-linearity and non-monotonic functions and models. This study illustrates its findings using the total sensitivity index which includes the main effect and parameter interactions. Quasi Monte Carlo is invoked as the sampling method.

The SWAT model can be regarded as an example of a complex, dynamic, over-parameterized environmental model, albeit in the hydrology domain. It has been used to simulate both water quantity and quality. It represents catchment processes based on spatially distributed soil type, weather variables, topography and land use. Multiple modules in SWAT handle hydrologic processes, weather conditions, erosion and nutrient processes. The SWAT model used here is based on previous studies by Leta et al. (2015) and Zadeh et al. (2015) for the Senne river basin in Belgium. This paper investigates how individual sources affect the results of a Sobol' global sensitivity analysis of the SWAT model. Sensitivity analyses are performed with different weather conditions and multiple objective functions, and the stability of the ranking of parameter sensitivity is discussed.

The objective functions used as illustration in this study are: the Nash-Sutcliffe Efficiency (NSE), the modified NSE (NSE*), NSE*Log, and NSE*combined. The study analyses the sensitivity indices and rank of the parameters for different weather conditions, using wet and dry calendar years selected from the five-year observation period. In case of the selected wet year, NSE and NSE* produce the same rank for parameter sensitivity. The objective functions NSE*Log and NSE*combined both return different sensitivity indices and rankings to NSE and NSE*, as they emphasize low flows and mid flows more than high flows. The SWAT parameter $C n 2$ (runoff curve number) becomes more influential in drier conditions whereas $C h \_K 2$ (effective hydraulic conductivity), for example, yields lower sensitivity indices for the dry year.

In addition, the study presents a visual comparison of the stability of relative sensitivities with the different sources using the estimated confidence intervals for different numbers of sampling runs. The SWAT model is generally insensitive to most parameters indicating that some of these parameters may require other conditions (i.e. a different catchment/climate) in order to be calibrated. This emphasises the need for GSA to determine which parameters are important for a given catchment when using very heavily parameterized models.

Keywords: Sensitivity analysis, SWAT, model parameters, Sobol' method, environmental modelling, identifiability 


\section{INTRODUCTION}

A sensitivity analysis (SA) can determine the most dominant parameters and inputs affecting predictions of a model, and can allow reduction in the number of calibrated parameters (Saltelli et al., 2004). Sensitivity analysis is one of the fundamental steps to help identify uncertainties relevant to the modelling objectives. It can contribute to simplifying over-parametrized models. However, SA may produce different results in accordance with several conditions, such as input data, objective functions, and the number of simulations undertaken when sampling the parameters and input ranges.

The Soil and Water Assessment Tool or SWAT (Arnold et al. 1996) is used in this paper to demonstrate some of the major effects on sensitivity that one needs to be aware of when trying to understand the behaviour of an environmental model. It can be regarded as an example of a complex, dynamic, over-parameterized environmental model, albeit in the hydrology domain. Many of the studies discussing sensitivity of SWAT model parameters employ sensitivity analysis as a preliminary process for model calibration. Muleta and Nicklow (2005) described an automatic approach for calibration of daily streamflow and daily sediment concentration values estimated using SWAT. Latin hypercube sampling was used to generate input data from the assigned distributions and ranges, and parameter estimation was performed using a genetic algorithm. Mulungu and Munishi (2007) showed that surface water model parameters such as Cn2 and Sol_K (Table 1) are the most sensitive and have more physical meaning.

Other papers provide an enhanced understanding of sensitivity to model inputs through consideration of the impact of climate conditions and flow regimes by comparing results for different catchments and different time periods. For example, Cibin et al. (2010) performed a sensitivity analysis for SWAT using the root mean square error (RMSE) or the Nash-Sutcliffe Efficiency (NSE) for two climatically different catchments, finding that the modelled streamflow time series shows variation in the sensitivity of parameters in different climatic settings, temporal scales and flow regimes. The limitation of such analyses is that the choice of objective function can also significantly affect the estimated sensitivities of the parameters.

In this paper, the Sobol' method (Sobol, 2001) is used to conduct a sensitivity analysis for parameters of a SWAT model of the Senne river basin in Belgium. The method estimates sensitivity indices which are caused by a single parameter or the interaction of two or more parameters (Saltelli et al., 2004). While the method has high computational demands compared to some methods, it is widely used for various applications because of its model independence and the ability to capture interaction effects.

The paper analyses quantitative sensitivities and qualitative rankings of parameter sensitivity derived using four objective functions: NSE, NSE*, NSE*Log and NSE*combined (Table 2). Further, the model's behaviour is evaluated separately for wet (2001) and dry (2004) years to investigate the effects of climate forcing on sensitivity and the lessons this may have for model calibration and acknowledging model limitations. The differences in the sensitivity indices and their ranking are discussed. Such results help in deciding which objective function(s) is more suitable to extract information from a calibration for the modelling purpose. The paper also presents a visual representation, in terms of confidence intervals obtained by bootstrapping, of the stability of sensitivity ranking with sample size for given objective functions and climate conditions.

\section{THE SWAT MODEL}

SWAT is a semi-distributed, physically based model that is used widely to predict water quantity and quality variables on a daily or sub-daily time step. SWAT models a catchment based on spatial information of soil type, weather variables, topography and land use. It divides a basin into sub-basins that are further divided into hydrological response units (HRUs). SWAT lumps all the similar soil and land use areas into a single response unit. It includes multiple modules to represent the following hydrological processes: interception, surface runoff, percolation, lateral subsurface flow, groundwater return flow, evapotranspiration and channel transmission losses. The behaviour of nutrients and pesticides can also be simulated (Arnold et al., 1996; ASCE, 1999).

This study is based on a previous study of the sensitivity analysis of the SWAT model for the river Senne in Belgium (Leta et al., 2015; Zadeh et al., 2015). The area of the catchment is some $642 \mathrm{~km}^{2}$ and was divided into 26 sub-basins and 194 HRUs. A total of 26 parameters (Table 1) of SWAT were used in a Sobol' sensitivity analysis employing Quasi Monte Carlo to sample the parameters. Quasi Monte Carlo is known to enhance the convergence rate compared to other sampling methods including basic Monte Carlo simulation and Latin Hypercube sampling (Tarantola et al., 2012).

The same SWAT model was used in this study, built using observations and climate variables from 1998 to 2005. The data from 1998 to 2000 is considered as a warm-up period. Similarly, results were produced for the 
period from 2001 to 2005 but in this paper results were generated for a relatively wet (2001) and dry (2004) calendar year in order to investigate the effects of climate forcing on sensitivity. Note that the wet year has higher rainfall and more persistent and higher baseflow than the dry year. In addition, our investigations also considered the effects of different objective functions and sample size on sensitivity.

Table 1. The 26 parameters of the SWAT model and their range in value

\begin{tabular}{|c|c|c|c|c|c|}
\hline Parameter & Definition & Unit & Process & Level & Range \\
\hline Slope & Average slope steepness & $\mathrm{m} / \mathrm{m}$ & $\begin{array}{l}\text { Lateral flow, } \\
\text { Sediment erosion }\end{array}$ & HRU & 0,1 \\
\hline Ch_K2 & $\begin{array}{l}\text { Effective hydraulic conductivity in main channel } \\
\text { alluvium }\end{array}$ & $\mathrm{mm} / \mathrm{h}$ & Routing & Sub-basin & 0,150 \\
\hline Alpha_Bf & Baseflow recession factor & days & Groundwater & HRU & 0,1 \\
\hline Rchrg_Dp & Groundwater recharge to deep aquifer & fraction & Groundwater & HRU & 0,1 \\
\hline GWQMN & $\begin{array}{l}\text { Threshold depth of water in shallow aquifer } \\
\text { for return flow to occur }\end{array}$ & $\mathrm{mm}$ & Groundwater & $\mathrm{HRU}$ & 10,500 \\
\hline Revapmn & $\begin{array}{l}\text { Threshold depth of water in the shallow aquifer for } \\
\text { 'Revap' }\end{array}$ & $\mathrm{mm}$ & Groundwater & HRU & 1,500 \\
\hline Gw_Delay & Groundwater delay & days & Groundwater & HRU & 1,60 \\
\hline Canmx & Maximum canopy index & & Evapotranspiration & HRU & 0,10 \\
\hline Gw_Revap & Groundwater 'Revap' coefficient & & Groundwater & $\mathrm{HRU}$ & $0.02,0.2$ \\
\hline Biomix & Biological mixing efficiency & & $\begin{array}{l}\text { Nitrogen cycle } \\
\text { Phosphorus cycle }\end{array}$ & HRU & 0,1 \\
\hline Epco & Plant evaporation compensation factor & & Evapotranspiration & HRU & $0.1,1$ \\
\hline SurLag & Surface runoff lag coefficient & & Surface runoff & Sub-basin & $0.5,10$ \\
\hline Smfmx & Maximum melt rate for snow & $\mathrm{mm} /{ }^{0} \mathrm{C} / \mathrm{day}$ & Snow & Sub-basin & 0,10 \\
\hline Smtmp & Snow melt base temperature & $\mathrm{mm} /{ }^{0} \mathrm{C} /$ day & Snow & Sub-basin & $-5,5$ \\
\hline Tlaps & Temperature laps rate & ${ }^{0} \mathrm{C} / \mathrm{km}$ & Temperature & Sub-basin & $-10,10$ \\
\hline Timp & Snow pack temperature lag factor & ${ }^{0} \mathrm{C}$ & Snow & Sub-basin & $-10,10$ \\
\hline Slsubbsn & Average slope length & M & $\begin{array}{l}\text { Concentration time, } \\
\text { Sediment erosion }\end{array}$ & HRU & 10,150 \\
\hline
\end{tabular}

\section{METHOD}

\subsection{Sobol' method}

The Sobol' method is a global sensitivity analysis (GSA) approach and is independent of the model to be analysed. The method is based on variance decomposition. It can handle non-linearity and non-monotonic functions and models. This study uses its total sensitivity index which includes the main effects and parameter interactions. Generally, GSA has to sample the entire parameter space using a random or systematic method. This is the reason why GSA has a high computational cost. For a highly parameterized, complex model such as SWAT, screening methods could be used to reduce the computational cost. However, screening methods only provide a relative rather than absolute indication of sensitivity. Examples of GSA methods are: Sobol' method, Fourier Amplitude Sensitivity Test (FAST), Morris method, Latin-Hypercube-One-factor-At-a-Time (LH-OAT). The Morris method and LH-OAT can be classified as screening methods (Nossent and Bauwens, 2012). 


\subsection{The objective functions}

We use four objective functions, as shown in Table 2 where $y_{o, i}$ is observed flow at time step $i, \bar{y}_{o}$ is the mean of observed flow, $y_{s, i}$ is simulated flow. The Nash-Sutcliffe efficiency (Nash and Sutcliffe, 1970) is one of the model performance scalar measures widely used in hydrology (known as the Coefficient of Determination in other fields). Because NSE uses the sum of squared residuals, its value tends to be affected most by high flows (Croke, 2009). Depending on model objectives, it may be advantageous to reduce this effect. The second objective function is a modified Nash-Sutcliffe Efficiency (Mathevet et al., 2006) denoted here by NSE* that produces a reduced range of values to the set $(-1,+1]$, rather $(-\infty,+1]$ as produced by the NSE. This aspect reduces the influence of large negative values without any change to the ranking of model performance for different parameter sets. The third is a log transformed NSE* denoted as NSE*Log, indicating that all the time series values used to calculate NSE* are $\log$ transformed. The NSE*Log statistic equilibrates the range of values of flow, with greater weight given to low flows than $\mathrm{NSE}^{*}$. A fourth alternative applied in the paper is $0.5 \mathrm{NSE}^{*}+0.5 \mathrm{NSE} * \mathrm{Log}$ denoted as NSE*combined, an evenly weighted average of the two previous objective functions.

Table 2. Objective functions invoked in the study

\begin{tabular}{|c|c|}
\hline Objective functions & Descriptions \\
\hline $\begin{array}{l}\text { The Nash-Sutcliffe } \\
\text { efficiency (NSE) }\end{array}$ & $N S E=1-\frac{\sum\left\{y_{o, i}-y_{s, i}\right\}^{2}}{\sum\left\{y_{o, i}-\overline{y_{o}}\right\}^{2}}$ \\
\hline $\begin{array}{l}\text { The modified Nash- } \\
\text { Sutcliffe efficiency } \\
\qquad\left(\text { NSE }^{*}\right)\end{array}$ & $N S E^{*}=\left\{1-\frac{\sum\left\{y_{o, i}-y_{s, i}\right\}^{2}}{\sum\left\{y_{o, i}-\overline{y_{o}}\right\}^{2}}\right\} /\left\{1+\frac{\sum\left\{y_{o, i}-y_{s, i}\right\}^{2}}{\sum\left\{y_{o, i}-\bar{y}_{o}\right\}^{2}}\right\}$ \\
\hline NSE*Log & $N S E^{*} \log =\left\{1-\frac{\sum\left\{\log \left(y_{o, i}\right)-\log \left(y_{s, i}\right)\right\}^{2}}{\sum\left\{\log \left(y_{o, i}\right)-\overline{\log \left(y_{o}\right)}\right\}^{2}}\right\} /\left\{1+\frac{\sum\left\{\log \left(y_{o, i}\right)-\log \left(y_{s, i}\right)\right\}^{2}}{\sum\left\{\log \left(y_{o, i}\right)-\overline{\log \left(y_{o}\right)}\right\}^{2}}\right\}$ \\
\hline NSE*combined & $\mathrm{NSE}^{*}$ combined $=0.5 \mathrm{NSE}^{*}+0.5 \mathrm{NSE}^{*} \mathrm{Log}$ \\
\hline
\end{tabular}

The four different objective functions in Table 2 were used to compute the Sobol' sensitivity indices for the period from 2001 to 2005 as well as both a wet year and a dry year. This allows investigation of the effect of climate on parameter sensitivity. The highest $60 \%$ of values in flow observations are extracted from each calendar year, fitted to the generalized extreme-value distribution, and from this a wet (2001) and dry (2004) calendar year were identified based on the mean annual discharge $\left(5.96 \mathrm{~m}^{3} \mathrm{~s}^{-1}\right.$ for 2001 and $3.19 \mathrm{~m}^{3} \mathrm{~s}^{-1}$ for 2004).

\section{RESULTS}

Table 3 shows the parameter sensitivity indices estimated using the alternative objective functions and wet and dry years. While the study evaluated the sensitivity of the 26 SWAT parameters, the table displays the results of the most sensitive 10 parameters.

\subsection{Sensitivity analysis in accordance with different climatic conditions}

There are significant differences in the ranking for wet and dry years when the NSE*Log and NSE*combined objective functions are used, indicating that weather and the resulting hydrograph can have significant impacts on the sensitivities of the parameters.

For the results with NSE* note that $C n 2$ (SCS curve number for moisture condition II) is the most sensitive parameter in both wet and dry years, as has been noted in previous research. $C h \_K 2$ (effective hydraulic conductivity in main channel alluvium) and $C h \_N 2$ (Manning coefficient for channel) can be categorized as parameters indicating channel properties. These parameters have higher sensitivity indices for the wet year. This shows that channel properties can be considered more sensitive parameters to improve model performance in wet conditions. Slope (average slope steepness) and Sol_K (soil conductivity) yield higher sensitivity indices in a dry year compared to a wet year. Increased sensitivity to Slope indicates that lateral flow becomes more influential on runoff in dry weather. Higher sensitivity to Sol_K indicates the movement of water through soil is more important in dry weather. The contribution of lateral flow or soil water to streamflow is likely to be increased in dry weather. In contrast, the parameters used for groundwater processes such as Alpha_Bf 
(baseflow recession factor), Rchrg_Dp (groundwater recharge to deep aquifer) and Gwqmn (threshold depth of water in shallow aquifer for return flow to occur) have lower sensitivity indices in dry weather. It seems that sensitivity analysis using different climatic forcings can reflect the variation in sensitivity of the streamflow to other hydrologic processes. In cases where climatic variation is significant in the record, model calibration on one mode of climate forcing is likely to be inadequate.

Table 3. Sobol' parameter sensitivities for each objective function and climate forcing

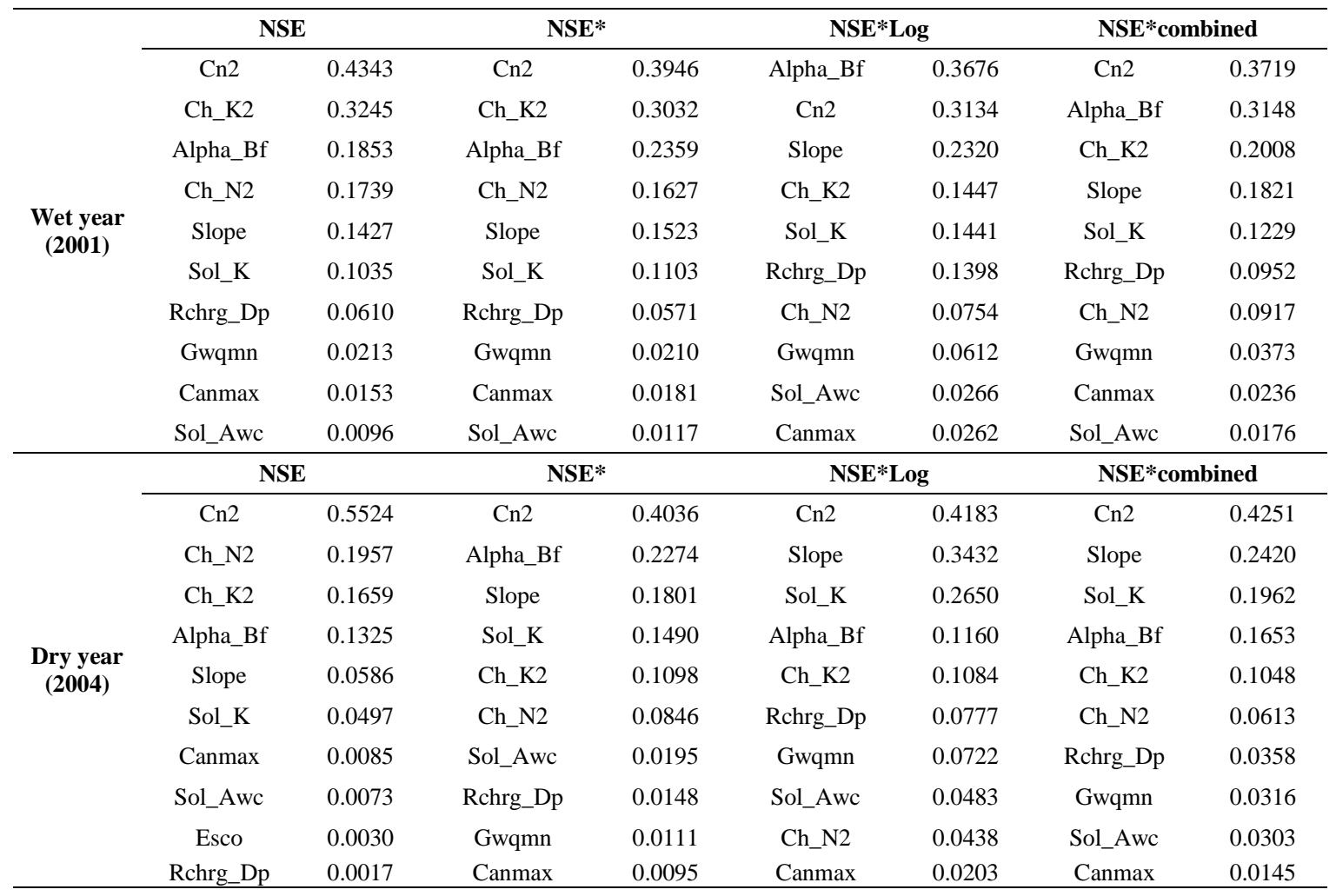

\subsection{Sensitivity analyses using different objective functions}

In the wet year, the sensitivity analyses with NSE and NSE* produce different values, but the same ordering of parameters, as expected given NSE* is a monotonic transformation of NSE. The results with NSE*Log or NSE*combined are quite different due to the increased weight given to low flows. Rchrg_Dp is more influential for low flows as it is one of the parameters which are used for groundwater processes. Slope and Sol_K have higher relative sensitivity when using NSE*Log or NSE*combined. In contrast, $C h \_K 2$ and $C h \_N 2$ have higher relative sensitivity for higher flow conditions (with NSE*). When calibrating or modifying a model, it is useful to know that NSE*Log or NSE*combined increases the sensitivity of parameters related to groundwater processes.

\subsection{Convergence and confidence intervals}

This section discusses the convergence of the sensitivity of each parameter, as indicated by confidence intervals estimated using 2000 bootstrap replicates. Figure 1 shows the sensitivity indices and confidence intervals. The upper panel displays the most sensitive 5 parameters and the lower panel show the $6^{\text {th }}$ to the $10^{\text {th }}$ most sensitive parameters. In this study, we focus on ordering of parameters, and consider sensitivities to be sufficiently precise when confidence bounds do not overlap. Thus Figure 1 shows that $C n 2$ and $C h \_K 2$ converge sufficiently at 2000 samples. $C h \_K 2$ and $A l p h a \_B f$ are ensured to converge sufficiently with 3000 samples. However, the $8^{\text {th }}, 9^{\text {th }}$ and $10^{\text {th }}$ parameters are not regarded as having converged with even 8000 samples, though one may not need this condvergence when a parameter is so insensitive.

\section{DISCUSSION AND CONCLUSIONS}

This study performed a Sobol' global sensitivity analysis of the Soil and Water Assessment Tool. The analysis was undertaken using daily data from 2001-2005 for the Senne river basin in Belgium in order to identify the effects of different objective functions, climate forcings and sample sizes on confidence in the sensitivities 


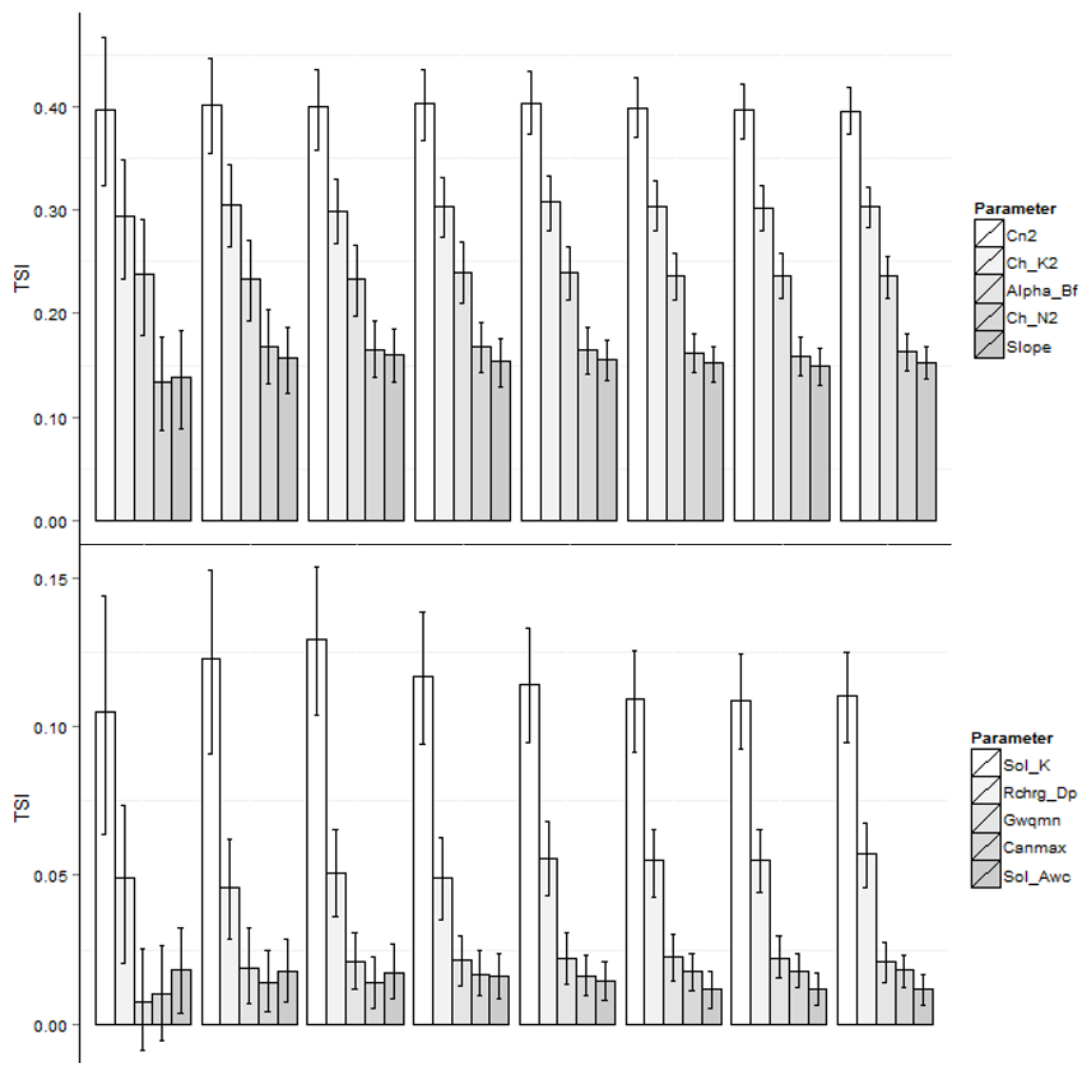

Figure 1. The sensitivity indices and confidence intervals in accordance with the number of samples/model runs in steps of 1,000 (NSE*, wet year)

generated. The more detailed findings can be considered as specific to the case study rather than a general phenomenon, and further study is warranted to appreciate the generality of them by employing different catchments and sufficiently longer observations.

On the other hand, it is clear that the choice of objective functions is critical when undertaking a GSA. The use of $\mathrm{NSE}^{*}$ reduced the influence of very poor models $(\mathrm{NSE}<<0)$, preventing them from dominating the calculated indices. The differences between NSE*, NSE*Log and NSE*combined reflect emphasis on different parts of the flow time series, namely high flows, low flows, and an equally weighted compromise. Different objective functions therefore provide different information about the model. It is also clear that there can be variations in sensitivity with long-term weather patterns as illustrated with the results for a wet versus dry year here. This emphasizes the problem with extrapolation of models to conditions that were not used in calibrating the model (e.g. climate change if the change is sufficiently large compared to the variability in the record analysed).

It also suggests the importance of understanding the characteristics of the data required to adequately estimate a model's parameters. This is a key concern of identifiability analysis, but is still an unsolved problem for very heavily parameterized hydrological models like SWAT. GSA using suitably chosen objective functions and data can contribute to this goal by determining which parameters are important for a given catchment, using that dataset and objective function. The SWAT model is generally insensitive to most parameters, indicating either that the parameter estimation is ill-conditioned (there is a wide range in sensitivities), or that some of these parameters may require data reflecting other conditions (e.g. a different catchment/climate) in order to be calibrated. However, it is not unlikely that some parameters are never sensitive whatever the catchment or climate forcing. Exhaustive testing on catchments from a wide range of hydroclimatologies would be a fruitful exercise as it could lead to further understanding of the SWAT model behaviour.

Better understanding of the SWAT model could lead to its ultimate modification to be more sensitive and perhaps less complex, with attendant benefits such as to reduced runtimes. If suitable data cannot be obtained, other changes may be possible. It may be possible to employ methods that make use of the insensitivity to facilitate uncertainty quantification (e.g. Null Space Monte Carlo, Keating et al. 2010). It may also be possible to fix or identify certain parameters with a more informed choice of objective function, but this would also 
require more in-depth analyses - using a simple accumulated objective function like those used here can completely mask parameters with sensitivity under particular conditions.

The study investigated the rate of reduction in the confidence intervals with increasing sample size to estimate the sample sizes required for sufficient confidence in the ranking of the more sensitive parameters. Here, sensitivity rankings are determined to have stabilised when confidence bounds no longer overlap. Further research could discuss different criteria to decide the convergence of sensitivity indices or ranks for the purpose of understanding identifiability and data requirements of highly parameterised models like SWAT.

Both technical advances and deep model understanding are required to help advance the reliability of hydrological modelling. Better understanding the use of sensitivity analysis methods is one key step in this direction.

\section{ACKNOWLEDGMENTS}

This work is supported by an Australian Postgraduate Award to the first author.

\section{REFERENCES}

Arnold, J. G., and Allen, P. M. (1996). Estimating hydrologic budgets for three Illinois watersheds. Journal of Hydrology, 176, 55-77.

ASCE, 1999. Anon. (1999). GIS Modules and Distributed Models of Watersheds. American Society of Civil Engineers, Reston, VA.

Cibin, R., Sudheer, K. P., and Chaubey, I. (2010). Sensitivity and identifiability of stream flow generation parameters of SWAT model. Hydrological Processes, 24.9, 1133-1148.

Croke, B.F.W. (2009). Representing uncertainty in objective functions: extension to include the influence of serial correlation. In Anderssen, R.S., R.D. Braddock and L.T.H. Newham (eds) 18th World IMACS Congress and MODSIM09 International Congress on Modelling and Simulation. Modelling and Simulation Society of Australia and New Zealand and International Association for Mathematics and Computers in Simulation, July 2009, pp. 3372-3378. ISBN: $978-0-9758400-7-8$. http://www.mssanz.org.au/modsim09/I7/croke.pdf.

Keating, E. H., Doherty, J., Vrugt, J. A., and Kang, Q. (2010). Optimization and uncertainty assessment of strongly nonlinear groundwater models with high parameter dimensionality. Water Resour. Res., 46(10).

Leta, O. T., Nossent, J., Velez, C., Shrestha, N. K., van Griensven, A., and Bauwens, W. (2015). Assessment of the different sources of uncertainty in a SWAT model of the River Senne (Belgium). Environmental Modelling \& Software, 68(2015), 129-146.

Mathevet, T., Michel, C., Andréassian, V., and Perrin, C. (2006). A bounded version of the Nash-Sutcliffe criterion for better model assessment on large sets of basins. Large Sample Basin Experiments for Hydrological Model Parameterization: Results of the Model Parameter Experiment - MOPEX, vol. 307. IAHS Publ., 211-219.

Muleta, M. K., and Nicklow, J. W. (2005). Sensitivity and uncertainty analysis coupled with automatic calibration for a distributed watershed model. Journal of Hydrology, 306(1), 127-145.

Mulungu, D. M., and Munishi, S. E. (2007). Simiyu River catchment parameterization using SWAT model. Physics and Chemistry of the Earth, Parts A/B/C, 32(15), 1032-1039.

Nossent, J., and Bauwens, W. (2012). Multi-variable sensitivity and identifiability analysis for a complex environmental model in view of integrated water quantity and water quality modeling. Water Science \& Technology, 65(3), 539-549.

Nossent, J., Elsen, P., and Bauwens, W. (2011). Sobol' sensitivity analysis of a complex environmental model. Environmental Modelling \& Software, 26(12), 1515-1525.

Saltelli, A., Tarantola, S., Campolongo, F., and Ratto, M. (2004). Sensitivity Analysis in Practice: A Guide to Assessing Scientific Models. John Wiley \& Sons, Ltd, Chichester.

Sobol, I. M. (2001). Global sensitivity indices for nonlinear mathematical models and their Monte Carlo estimates. Mathematics and computers in simulation, 55(1), 271-280.

Tarantola, S., Becker, W., and Zeitz, D. (2012). A comparison of two sampling methods for global sensitivity analysis. Computer Physics Communications, 183(5), 1061-1072.

Zadeh, F. K., Sarrazin, F., Nossent, J., Pianosi, F., Griensven A. V., Wagener, T., and Bauwens, W. (2015). Comparison of the PAWN and Sobol' sensitivity analysis methods for a highly-parameterized hydrological model using SWAT. Proceedings of the $36^{\text {th }}$ IAHR World Congress, The Hague, the Netherlands. 